\title{
Economical Analysis of High Volume Flexible Manufacturing System for Agile Manufacturing
}

\author{
Susumu Fujii, Hiroshi Morita, Masahiro Imada, Hiroyuki Iio \\ Dept. of Computer and Systems Engineering, Kobe University \\ Nada, Kobe 657-8501 Japan. \\ e-mail: \{fujii, morita\}@seg.kobe-u.ac.jp \\ Yasuto Tatsuta \\ Mazda Motor Corporation, Hiroshima, Japan \\ Yoshiharu Takata \\ Yamazaki Mazak Corporation, Aichi, Japan
}

\begin{abstract}
We focus on the economical aspect of a high-volume flexible manufacturing system (HV-FMS) for agile manufacturing. We develop a model to estimate the economical behavior of HV-FMS and flexible transfer line. Under several demand patterns which have been generated from historical data, both models are simulated to evaluate the effectiveness of HV-FMS from an economical view point.
\end{abstract}

High-volume FMS, agile $\begin{gathered}\text { Keywords } \\ \text { manufacturing, economical analysis }\end{gathered}$

\section{INTRODUCTION}

In recent manufacturing systems, it is necessary to process many kinds of products to meet the increasing and varying demand of customers. As a result, the life cycle of products is becoming shorter and the agility in manufacturing is strongly required to respond quickly to the changes not only in the variety but also in the quantity of products without losing the high quality. The flexible manufacturing 
system (FMS) has been introduced to improve the productivity in manufacturing many kinds of products aiming at medium production capacity in the whole. Because of the frequent changes in the kinds and the volume of workpieces even in the mass production such as automobile industries, it is becoming necessary to develop manufacturing systems not only with flexibility but also large production capacity, which have provided by dedicated manufacturing systems such as flexible transfer lines (FTL).

To cope with such requirement, we propose a new manufacturing system, named a high volume flexible manufacturing system (HV-FMS), for agile manufacturing which attains the high flexibility for high volume production and demonstrated the effectiveness of the parallel-serial arrangement of machining cells by simulation study (Fujii et al (1998)). In this study, we focus on the economical aspect to HV-FMS. We develop a model to estimate the economical behavior of HV-FMS for long term. For comparison, a similar model for FTL is also developed. Under several demand patterns generated from the historical data, both models have simulated to evaluate the effectiveness of HV-FMS from an economical view point.

\section{HIGH VOLUME FLEXIBLE MANUFACTURING SYSTEM}

HV-FMS is designed for large volume parts production using a particular parallel-serial arrangement of machining cells to achieve high flexibility and high productivity. Figure 1 shows a typical workpiece flow. The basic unit is a machining cell which has a loading buffer, a machining station and an unloading buffer. Each cell forms an independent subsystem and the total system is their integration. The machining process is divided into several processing groups, each of which consists of same machining cells and is arranged in a series. A workpiece is fixed on a specific palette and can be processed by any machining cell in the group. An

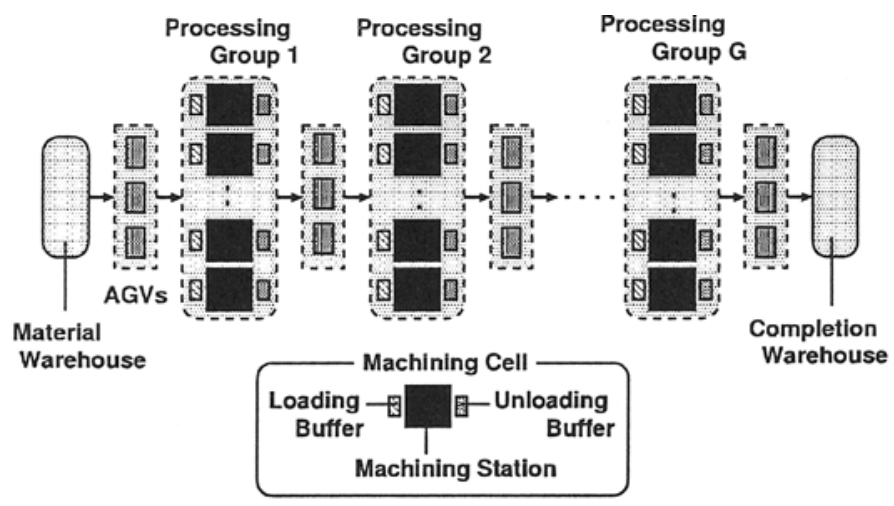

Figure 1 Configuration of HV-FMS. 


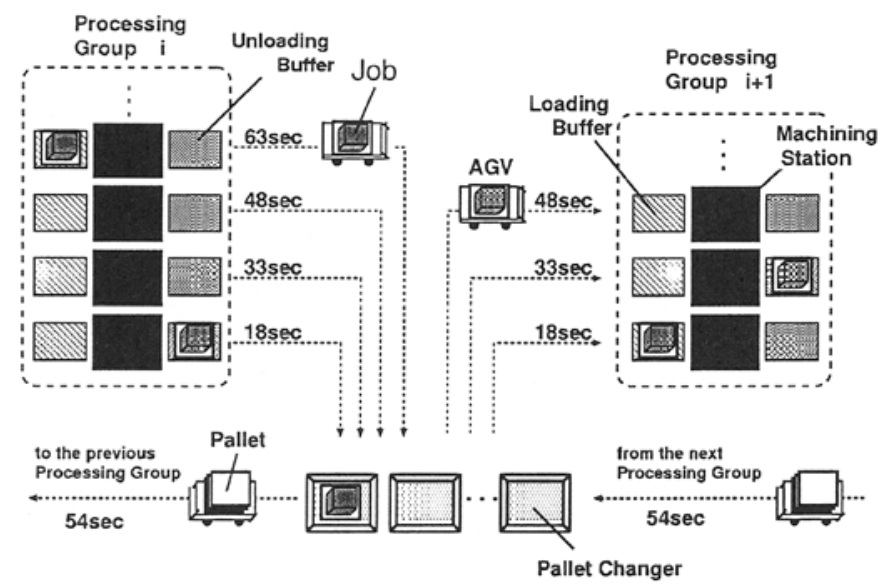

Figure 2. Transportation system and transfer time

automated guided vehicle (AGV) transports the workpiece between machining cell and palette changer. The machining cell to process the workpiece is selected according to dispatching rule such as the earliest available order. Figure 2 shows an illustration of transportation system and the transfer times predetermined for simulation study.

We consider HV-FMS which consists of $G$ processing groups to process $N$ kinds of products. The processing group has some machining cells with the same specification, each of which has the necessary processing tools in its machining station. The role of palette changer is to put off the workpiece from the palette used at the previous processing group and to put on it to a new palette used at the next processing group. Between processing groups, we assign appropriate number of AGVs, palettes and palette changers.

In spite of a bit of higher failure rate, the parallel arrangement of machining cells with the same specification realizes a continuous processing by using alternative machining cells when machine failure occurs. HV-FMS is robust for the machine failure and keeps the high productivity.

\section{ECONOMIC MODEL}

We make an economic model for $Y$ years. The net profit $P$ is obtained from total sales substituting an operation cost, a cost for equipment change, a cost for equipment expansion and an initial investment, that is,

$$
P=\sum_{j=1}^{Y} \frac{(1+u)^{j} p^{\prime} V_{j}}{(1+r)^{j}}-\left(\sum_{j=1}^{Y} \frac{C_{j}+F_{j}+A_{j}}{(1+r)^{j}}+C_{0}\right)
$$

where $r$ is an interest rate, $p$ is a sales price vector of products and $u$ is its inflation 
rate, $V_{j}$ is a production amount vector, $C_{j}$ is an operation cost, $F_{j}$ is a cost for equipment expansion, $A_{j}$ is a cost for equipment change of $j$ th year, and $C_{0}$ is an initial investment. Note that $F_{j}$ is brought to respond the change of product mix and additional equipment to make up for a lack of production.

The initial investment of HV-FMS is estimated by

$C_{0}=c_{M} n_{M}+c_{T} n_{T}+c_{V} n_{V}+c_{C} n_{C}+c_{P} n_{P}+Z$

where costs and total assigned numbers of machining cell, processing tool, AGV, palette changer and palette are $c_{M}, c_{T}, c_{V}, c_{C}, c_{P}$ and $n_{M}, n_{T}, n_{V}, n_{C}, n_{P}$, respectively, and $Z$ is miscellaneous cost for system construction. The operation cost $C_{j}$ for $j$ th year is estimated by the sum of costs of materials $(1+v)^{j} q^{\prime} M_{j}$, personnel $L_{j} W$, inventory $b_{j} c_{S}$, repair $d_{j} c_{R}$, energy $E_{j}$, processing tool $T_{j}$, supplement materials $H_{j}$ and preservation $U_{j}$, that is,

$C_{j}=(1+v)^{j} q^{\prime} M_{j}+L_{j} W+b_{j} c_{S}+d_{j} c_{R}+E_{j}+T_{j}+H_{j}+U_{j}$

where $q$ is a purchased cost vector of materials and $v$ is its inflation rate, $L_{j}$ is a personnel expense per worker with overtime work expense, $W$ is number of workers, $b_{j}$ is an amount of inventory, $c_{S}$ is an inventory cost per unit, $d_{j}$ is a number of failure and $c_{R}$ is a repair expense. Note that the personnel expense within prescribed working hours is fixed, and for the overtime work is proportional to the overtime working hours. The overtime work is done when we can not produce the necessary amount of production within the prescribed working hours.

FTL is a serial production line with many machining cells, which is illustrated in Figure 3. A workpiece is transported by a conveyer. The initial investment and the operation cost are estimated by a similar manner to HV-FMS.

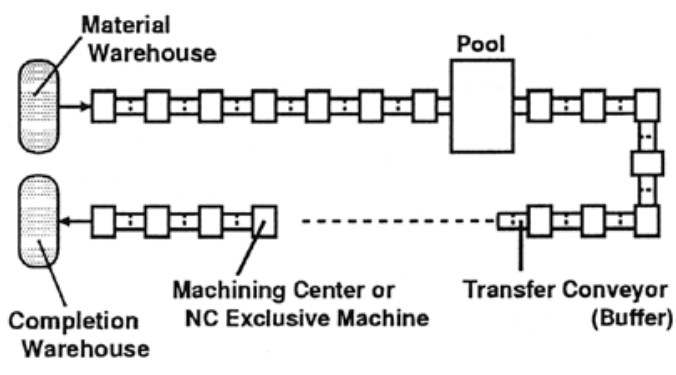

Figure 3. Configuration of FTL.

\section{OPERATION SYSTEM AND COST ELEMENTS}

We consider the production system for cylinder heads of automobile parts. We describe the specification of simulation model and numerical data for economical analysis for HV-FMS and FTL.

\subsection{Operation System}

$H V-F M S$

The specification of HV-FMS is as follows. 
(1) Processing equipment : There are five processing groups OP10, OP20, OP30, OP40, OP50. The cycle time is set to 48 seconds to produce 30,000 products per month. The machining cell to process the workpiece is selected randomly from available machining cells, but when there is no available machining cell, it is selected from machining cells with empty loading buffer. The time between failure (TBF) is exponentially distributed with the mean of 430 hours, and the time to repair (TTR) is log-normally distributed with the mean of 3.1 hours and the standard deviation of 3.3 hours.

(2) Transportation equipment : One AGV can transport one workpiece at a time. Seven AGVs are assigned between processing groups, that is, three are for transportation from previous machining cell to palette changer, other three are from palette changer to next machining cell, and one is to transport palettes to previous palette changer. Note that three AGVs and four AGVs are assigned between material warehouse and the first processing group and last processing group and completion warehouse, respectively. The transportation times are shown in Figure 2. The AGV to transport workpiece is randomly selected from available AGVs. We ignore the conflict and failure of AGVs.

(3) Palette changer : We assign four palette changers between processing groups and two palette changers between material warehouse and the first processing group as well as last processing group and completion warehouse. Total number of palettes is 50, which are allocated to each processing group according to the initial product mix. The reallocation of palettes is done by exchanging the jig on the palette to respond the change of product mix. The palettes are transported to the palette changer in the previous processing group every five palettes. The time for palette exchange is set to 3 minutes.

(4) Processing time and number of machining cells : We process six types of workpieces $\left(J_{A}, J_{B}, J_{C}, J_{D}, J_{E}, J_{F}\right)$ with derivatives. The processing time at each processing group is determined for every types of workpieces shown in Table 1. The number of machining cells is decided by the ratio of weighted mean of processing time to cycle time. The initial product mix is $\left(\mathrm{J}_{A}, \mathrm{~J}_{B}, \mathrm{~J}_{C}, \mathrm{~J}_{\mathrm{D}}, \mathrm{J}_{\mathrm{E}}\right.$, $\left.\mathrm{J}_{\mathrm{F}}\right)=(0.386,0,0.365,0,0.249,0)$. Then the weighted processing time for each processing group is $(5.695,4.827,6.959,3.719,7.270)$. The necessary number of machining cells for each processing group is obtained by 7, 6, 9, 5 and 9, respectively, by rounding to integer.

Table 1. Processing times (second)

\begin{tabular}{|l|r|r|r|r|r|}
\hline & \multicolumn{1}{|c|}{ OP10 } & \multicolumn{1}{|c|}{ OP20 } & \multicolumn{1}{|c|}{ OP30 } & \multicolumn{1}{|l|}{ OP40 } & \multicolumn{1}{|c|}{ OP50 } \\
\hline $\mathrm{J}_{\mathrm{A}}, \mathrm{J}_{\mathrm{B}}$ & 344 & 329 & 515 & 240 & 480 \\
\hline $\mathrm{J}_{\mathrm{C}}, \mathrm{J}_{\mathrm{D}}$ & 276 & 301 & 351 & 194 & 240 \\
\hline $\mathrm{J}_{\mathrm{E}}, \mathrm{J}_{\mathrm{F}}$ & 434 & 212 & 364 & 240 & 480 \\
\hline
\end{tabular}

(5) Tool magazine : Since each machining cell has all necessary processing tools at each processing group, there is no setup time to process many kinds of workpieces. The number of processing tools for each processing group is 79, $108,48,0,2$, respectively. Note that OP40 has no processing tool. 
(6) Equipment change : When new type of workpiece or derivatives are added, it is necessary to stop the whole production system for the equipment change. The equipment change takes one day for minor derivative, and three days for major derivative or new workpiece type. For the change of product mix, the palettes should be reassigned according to new product mix, by exchanging the jig on palette, not by adding new palette.

FTL

It is difficult for FTL to process many types of workpieces at one production line. We provide three production lines to process the same amount and the same kinds of products to HV-FMS. The specification of FTL is as follows.

(1) Processing equipment : There are 15 processes for each production line. The cycle time is set to 173 seconds to produce 10,000 products per month. Each line has two buffers with capacity of 200. The TBF of each process is exponentially distributed with the mean of 650 hours, and the TTR is log-normally distributed with the mean of 3.4 hours and the standard deviation of 6.5 hours. The processing time and the transfer time to the next process are given from historical data.

(2) Transportation equipment: The workpieces are transported by using a conveyer, which has role of buffer with capacity of two.

(3) Setup time : A setup is required to change the type of processing workpieces. The setup time is 90 minutes for the change to the major derivatives or new workpiece type.

(4) Equipment change : When we change the production line, it takes five days for minor change, and ten days for medium or major change.

\subsection{Cost Elements}

Common elements are set to the followings. $Y=15$ years, $i=0.8 \%$ per month, $u=0 \%$, $\nu=0 \%, p=10,000$ yen for all products, $q=4,000$ yen for all products, $c_{R}=100,000$ yen, $E=200$ yen per product, $T=200$ yen per product, $H=50$ yen per product and $U=100,000$ yen per month. The personnel expense is estimated as the prefixed working time is 480 hours per month, maximal overtime work is 218 hours per month, the fixed personnel expense is 500,000 yen per person per month and overtime work expense is 3,600 yen per hour. We set $S=0$, since we do not produce excess products even if it is in prefixed working time.

Table 3. Cost elements of HV-FMS and FTL. (million yen)

\begin{tabular}{|c|l|l|}
\hline & HV-FMS & FTL \\
\hline Initial investment & 3,600 & $1,200 \times 3$ lines \\
\hline Equipment change & & \\
Large change & 165 & 385 \\
Small change & 15 & 10 \\
Jig exchange & 1 & n.a. \\
\hline Number of workers & 9 & $9 \times 3$ lines \\
\hline
\end{tabular}


The cost elements for each of HV-FMS and FTL are summarized in Table 3, where the items of initial investment for HV-FMS are $c_{M}=31.2, c_{T}=0.1, c_{V}=6, c_{C}=10$, $c_{P}=3, Z=1151.7$ million yen .

\section{ECONOMICAL ANALYSIS}

\subsection{Demand Models}

Let the production amount at each term be the predicted demands shown in Figure 4, which is also generated from the historical data. In each type of workpieces, there are two kinds of derivatives, that is, one is a minor derivative and the other is a major derivative. Figure 5 shows demands for workpiece type $\mathrm{J}_{\mathrm{C}}$ for example. $\mathrm{C} 12$ is a minor derivative from $\mathrm{C} 11$, and $\mathrm{C} 21$ is a major derivative from $\mathrm{Cl}$ 's.

\subsection{Economical Analysis}

Using the models developed in the previous sections, we estimate the net profits for HV-FMS and FTL under the above demand. The workpieces to be processed are various kinds and mass volume, in fact, there are six types of workpieces and their 18 derivatives, and totally 30,000 products per month. Figure 6 shows the cash flow for HV-FMS and FTL, which implies that HV-FMS brings much higher net profit than FTL because of the low cost for equipment change and personnel. Moreover, the pay-back period of investment is 41 months for HV-FMS and 48 months for FTL. HV-FMS can collect the investment much faster than FTL.

Next, we investigate the effectiveness against the change of production amounts. Figures 7 and 8 show the cash flows, when the amount of demands of $\mathrm{J}_{\mathrm{A}}$ and $\mathrm{J}_{\mathrm{B}}$ are $20 \%$ increased and $20 \%$ decreased, respectively. It is clear that HV-FMS has a obvious benefit from additional production, however, FTL has only nominal benefit. On the other hand, when the demands are decreased, there is no significant difference between HV-FMS and FTL, that is, the net profits decreases for both systems.

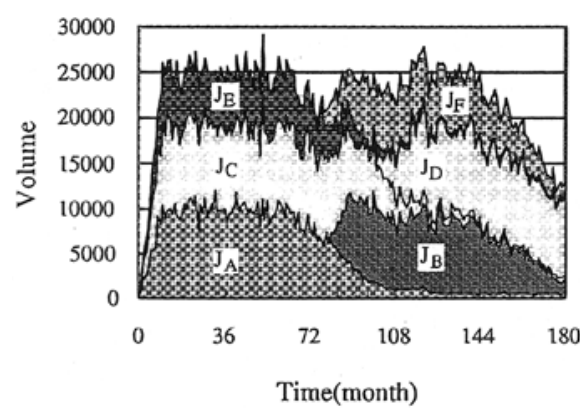

Figure 4. Demand model.

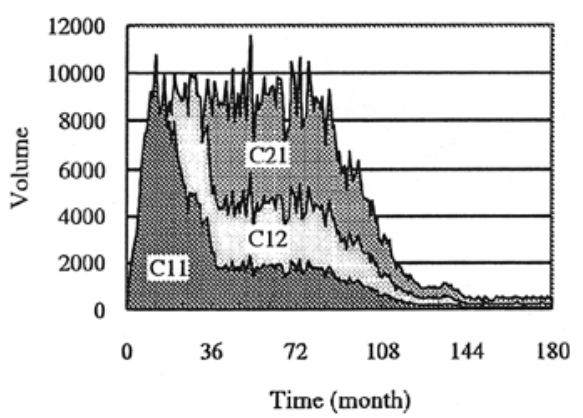

Figure 5. Demand for Jc. 

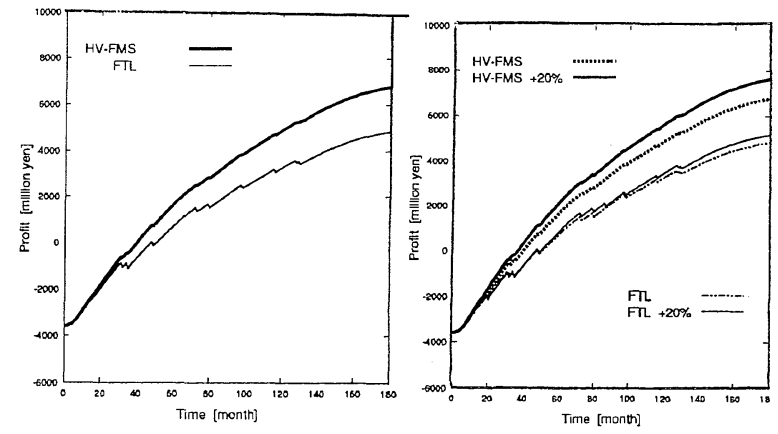

Figure 6. Cash flow
Figure 7. Cash flow for increasing demand

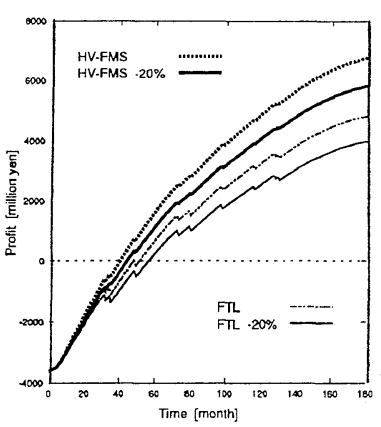

Figure 8. Cash flow for decreasing demand.

\section{CONCLUSION}

We reviewed the configuration of HV-FMS. The factors considered to develop an economical model were discussed, yielding the proposed model for economical analysis. After presenting a similar model for FTL, we demonstrated the economical effectiveness of HV-FMS by simulation runs based on several demand patterns. We can conclude that HV-FMS is an economically effective production system, which has high flexibility to the change of products and high productivity.

\section{REFERENCES}

Fujii, S., Morita, H., Tatsuta, Y. and Takata, Y. (1998) A Basic Study on High Volume Flexible Manufacturing System for Agile Manufacturing, in Advances in Production Management Systems (N. Okino et al. Eds.), pp. 67-78, Chapman \& Hall, London.

Kidd, P. T. (1995) Agile Manufacturing, forging New Frontiers, Addison-Wesley Publishers, Reading.

Raouf, A. and Ben-Daya, M., eds. (1995) Flexible Manufacturing Systems: Recent Developments, Manufacturing Research and Technology 23, Elsevier Science B. V., Amsterdam.

\section{BIOGRAPHY}

Susumu Fujii is Professor, Hiroshi Morita is Associate Professor, Masahiro Imada and Hiroyuki lio are Mater course students all at Department of Computer and Systems Engineering, Kobe University. Yasuto Tatsuta is General Manager at Product and Production Engineering Administration Office, Mazda Motor Corporation. Yoshiharu Takata is Assistant Leader at Development \& Design Division, Yamazaki Mazak Corporation. 